\title{
Clinical Analysis of risk factors for 30-day mortality in type A acute aortic dissection: a single-center study from China
}

\author{
mingxing xie ${ }^{1}$, Hongliang Yuan ${ }^{1}$, Yuman $\mathrm{Li}^{1}$, Wenqian $\mathrm{Wu}^{1}$, Yongxing Zhang ${ }^{1}$, and Manwei \\ $\mathrm{Liu}^{2}$ \\ ${ }^{1}$ Affiliation not available \\ ${ }^{2}$ Wuhan Union Hospital Library
}

May 10, 2021

\begin{abstract}
Objective: This study was conducted to evaluate pre- and intraoperative risk factors for 30-day mortality in patients with acute type A aortic dissection (ATAAD). Methods: Three hundred thirteen patients with ATAAD who underwent emergency surgery (264 men and 49 women; mean age, $48 \pm 10$ years) were enrolled in our study. Preoperative and operative risk factors for death were presented. Multivariable analysis was performed to identify the influence of varying factors on 30-day mortality. Results: Overall, 32 patients $(10.2 \%)$ died within 30 days. Compared with the surviving group, the deceased patients were more likely to have tachycardia, elevated serum potassium levels, moderate to severe pericardial effusion, suprasternal branch involvement, myocardial ischemia, and lower-extremity ischemia. Regarding factors related to surgery, the duration of surgery and cardiopulmonary bypass and concomitant procedures of coronary artery bypass graft(CABG) were greater in patients who died. In multivariate analysis, independent risk factors were longer duration of surgery (odds ratio [OR]: 4.5, $\mathrm{p}=0.001$ ) and cardiopulmonary bypass (OR: $5.3, \mathrm{p}=0.001$ ), moderate to severe pericardial effusion (OR: $3.3, \mathrm{p}=0.017$ ), suprasternal branch involvement (OR: 4.9, $\mathrm{p}=0.002$ ), and lower-extremity ischemia (OR: 7.6, pi0.001). Conclusions: Lower-extremity ischemia and suprasternal branch involvement have the poorest outcomes. Moderate to severe pericardial effusion could influence the outcome. Shorter duration of surgery is associated with better outcomes. Key Words: acute type A aortic dissection, surgery, mortality, risk factors
\end{abstract}

\section{Clinical Analysis of risk factors for surgery mortality}

\section{in type A acute aortic dissection: a single study from China}

Hongliang Yuan ${ }^{1,2 \#}, \mathrm{MD}$, Yuman $\mathrm{Li}^{1,2 \#}, \mathrm{MD}$, PHD, Wenqian $\mathrm{Wu}^{1,2}, \mathrm{MD}$, PHD, Yongxing Zhang ${ }^{1,2}$, MD, Manwei $\mathrm{Liu}^{1,2}$, MD, PHD, Mingxing Xie ${ }^{1,2^{*}}, \mathrm{MD}$, PHD

Department of Ultrasound, Union Hospital, Tongji Medical College, Huazhong University of Science and Technology, Wuhan 430022, China;

Hubei Key Laboratory of Molecular Imaging, Union Hospital,Tongji Medical College, Huazhong University of Science and Technology, Wuhan 430022, China.

\#Drs Yuan and Li contributed equally to this work.

Corresponding Author:

Mingxing Xie, MD, PhD; Department of Ultrasound, Union Hospital, Tongji Medical College, Huazhong University of Science and Technology, 1277\# Jiefang Ave, Wuhan 430022, China. Tel: 86-27-85726430; Fax: 86-27-85726386. E-mail address:xiemx@hust.edu.cn 
The data that support the findings of this study are available from the corresponding author upon reasonable request.

\begin{abstract}
Objective : This study was conducted to evaluate pre- and intraoperative risk factors for mortality in patients with Acute type A aortic Dissection (ATAAD).

Methods : 313 patients (264 men and 49 women; mean age, $47.9 \pm 9.74$ years) were enrolled, who underwent emergency surgery. Preoperative and operative risk factors for death were presented. Multiple analysis were performed to identify the influence of factors on 30-day mortality.

Results: Overall, 32 patients $(10.2 \%)$ died within 30 days. When compared with survived team, the deceased patients are tachycardia, have more elevated serum potassium levels, moderate to severe pericardial effusion, suprasternal branch involvement, myocardial ischemia, and lower-extremity ischemia. As for factors related to surgery, the duration of operation, cardiopulmonary bypass time, concomitant procedure of CABG were greater among patients who died. On multivariate analysis, independent preoperative risk factors were longer duration of operation(OR 4.5, $\mathrm{p}=0.001)$ and cardiopulmonary bypass time(CPBT) $(\mathrm{OR} 5.3, \mathrm{p}=0.001)$, moderate to severe pericardial effusion(OR 3.3, $\mathrm{p}=0.017)$, suprasternal branch involvement $(\mathrm{OR} 4.9, \mathrm{p}=0.002)$, and lower-extremity ischemia(OR 7.6,pi0.001). Shock or cardiac tamponade were not risk factors.
\end{abstract}

Conclusions: Lower-extremity ischemia and suprasternal branch involvement have the poorest outcome. Moderate to severe pericardial effusion may influence the outcome. Shorter duration of operation is associated with better outcomes.

Key Words : Acute type A aortic dissection, Surgery, mortality, risk factors

\title{
Introduction
}

Acute type A aortic dissection (ATAAD) is a life-threatening cardiovascular condition that requires emergency surgery. Recently, the incidence of ATAAD has been increasing with the increasing incidence and poor control of hypertension in patients (1). With the great progress in various imaging technologies, our understanding of ATAAD has become clearer than it was previously. With the rapid development of materials technology and improved outcome expectations, the therapeutic strategies and concepts for ATAAD are constantly being updated. Therefore, further analysis and recognition of this critical and severe disease are needed to improve the clinical prognosis. The purpose of this study was to identify risk factors for in-hospital mortality in patients with ATAAD.

\section{Methods}

\section{Patients}

Three hundred twenty-five patients with ATAAD at Union Hospital from February 2012 through February 2017 were studied. All patients were diagnosed by surgery or imaging examinations, such as computed tomography angiography (CTA), magnetic resonance imaging (MRI) or echocardiography. Patients with chronic type A aortic dissection $(n=4)$ who were treated with intracavitary interventional therapy $(n=6)$ or who died after conservative treatment $(n=2)$ were excluded. Finally, a total of 313 patients (of whom 264 were male and 49 female; mean age, 48 years; range 22 to 70 years) were analyzed.

\section{Study parameters}

To investigate the influence of pre- and intraoperative variables on 30-day mortality, various parameters deemed relevant were tested in the statistical analysis (tables 1-2). Preoperative parameters covered demographics, echocardiographic measurements, vessel involvement and laboratory results, while operative parameters included procedure type and duration of procedure. The 30-day mortality included any death occurring from the intraoperative period through the 30th postoperative day. The study was approved by the institutional ethics board of Union Hospital Tongji Medical College, Huazhong University of Science and Technology. 


\section{Statistical analysis}

The statistical analyses were performed with SPSS software, version 21.0 (SPSS Inc., Chicago, IL, USA). The summary statistics are presented as frequencies and percentages for categorical values, as the means and standard deviations for normally distributed continuous values and as medians with quartiles for nonnormally distributed continuous variables. Comparisons between the groups were performed with Student's unpaired $\mathrm{t}$ test or the $\chi^{2}$ test. Probability values of less than 0.05 were considered to be statistically significant. The influence of different parameters on 30-day mortality was analyzed using multiple logistic regression analyses with backward elimination. The results of the logistic regressions are presented as odds ratio (OR) with confidence intervals. The possible risk factors (demographics, echocardiographic measurements, vessel involvement, laboratory results, procedure time and extracorporeal circulation time) included in the logistic regression analysis were analyzed using receiver-operating characteristic (ROC) curves to obtain diagnostic cutoff values, as well as specificity and sensitivity.

\section{Results}

In this study, 313 surgically treated ATAAD patients were included. Of these patients, $32(10.2 \%)$ died within 30 days.

Compared to the nonsurvivor group, the survivor group had lower heart rates and blood potassium levels. Moderate or severe pericardial effusion, suprasternal branch involvement, myocardial ischemia and lower limb ischemia were less common in patients who survived than in those who did not. However, there were no significant differences in age; blood pressure, blood leukocytes, neutrophils and platelet counts on admission; serum creatinine; ascending aorta diameter; left ventricular ejection fraction; degree of aortic regurgitation; dissection involving the ascending aorta and aortic arch; or lining breach position between the two groups (table 1).

Regarding the intraoperative factors, the durations of procedure and cardiopulmonary bypass time (CPBT) of the patients in the nonsurvivor group were significantly longer than those in the survivor group. Coronary artery bypass graft $(\mathrm{CABG})$ was performed more frequently in the nonsurvivor group than in the survivor group. There were no statistically significant differences between the two groups in terms of aorta occlusion time, cessation time, cerebral perfusion time, ascending aorta replacement, aortic valve replacement, half or whole arch replacement, stenting rhinoplasty or sinus molding (table 2).

ROC curves were used to determine the optimal truncation values for operation time, extracorporeal circulation time, admission heart rate and blood potassium level to identify early mortality in ATAAD patients (table 3, figure 1). ROC analysis revealed that duration of surgery $>9.5 \mathrm{~h}$ [area under the curve (AUC): 0.73], CPBT $>227$ min (AUC: 0.72 ), heart rate $>82$ beats/min (AUC: 0.65 ) and plasma potassium $>3.8$ mmol/l (AUC: 0.71 ) were more likely to be associated with 30-day mortality in patients with ATAAD.

Multivariable logistic regression analysis revealed that operation time $>9.5 \mathrm{~h}$ (OR: $4.5 ; 95 \%$ confidence interval [CI]:1.9-10.6; $\mathrm{p}=0.001$ ), a CPBT $>227$ min (OR: 5.3; 95\% CI:2.1-13.9; $\mathrm{p}=0.001$ ), moderate to severe pericardial effusion (OR: 3.3; 95\% CI: 1.1-9.6; $\mathrm{p}=0.017$ ), supraventricular branch involvement (OR: 4.9; $95 \%$ CI:1.8-13.4; $\mathrm{p}=0.002$ ), and lower limb ischemia (OR: 7.6; 95\% CI: 2.6-22.2; $\mathrm{p}<0.001$ ) were independent risk factors for early postoperative mortality (table 4 ).

\section{Comment}

ATAAD is a critical illness with high mortality. In this study, 32 patients died within 30 days after surgery $(10.2 \%)$, and the surgical mortality rate was lower than in previous studies. However, the surgical mortality of ATAAD remains unacceptable, so it is necessary to summarize the risk factors for surgical mortality that have been identified in patients with ATAAD in recent years. Large multicenter studies have analyzed the risk factors of mortality in ATAAD patients. The German Registry for Acute Aortic Dissection type A detected that longer operating times and comatose and resuscitated patients had the poorest outcomes (2). The International Registry of Acute Aortic (IRAD) concluded that surgical mortality was mainly related to unstable patients with preoperative organ malperfusion and time to surgery (3). However, a study from 
China showed that in-hospital adverse outcomes were associated with older age, presentation of lower limb symptoms prior to surgery and long CPBT (4). This retrospective study analyzed various parameters over 5 years and identified some risk factors for surgical mortality in ATAAD, with benefits for postoperative outcomes. Our findings showed that longer durations of surgery and CPBT, moderate to severe pericardial effusion, suprasternal branch involvement, and lower-extremity ischemia were independent risk factors for early postoperative mortality.

The multifactorial analysis in this study indicated that supraventricular branch involvement was a risk factor for postoperative mortality in patients with ATAAD. The extent of ATAAD involvement largely determined the preoperative state of the patients, and the postoperative outcome was greatly affected. In particular, involvement of the upper arch branch and the blood supply to the brain resulted in poor cerebral perfusion. In addition, anastomosis of branch vessels was required during surgery in these patients, prolonging the time of extracorporeal circulation and operation, which further affected cerebral perfusion. Some studies have shown that cerebral ischemia is a risk factor for in-hospital and postoperative mortality $(5,6)$. To prevent irreversible ischemic damage to the nervous system, it is of vital importance to administer timely and effective drug treatments and surgical treatments for patients who are unconscious or in a coma.

In this study, the proportion of patients with lower limb ischemia was significantly greater in the nonsurvivor group than in the survivor group. Multiple regression analysis showed that lower limb ischemia was a risk factor for early mortality in ATAAD patients, as in previous studies $(7,8)$. Poor perfusion of the lower extremities can lead to serious complications. ATAAD patients with lower limb ischemia present with no pulse or dysplasia and should be treated with appropriate attention. Uchida et al. improved the blood supply by draining the brachial arterial blood to the ischemic lower limb arteries, thereby significantly improving symptoms (9). However, Preece et al. conducted a literature analysis and concluded that inferior-limb ischemic artery reperfusion before aortic repair increased intraoperative mortality in ATAAD patients (10). Therefore, whether preoperative ischemic arterial perfusion of the lower extremities before aortic repair can improve the prognosis of surgery remains to be analyzed with additional sample data.

The duration of extracorporeal circulation was significantly longer in the nonsurvivor group than in the survivor group. The multivariable analysis showed that the duration of surgery and the duration of extracorporeal circulation were risk factors for early postoperative mortality. Our findings are consistent with previous studies (11). A long operation time reflects the severity of ATAAD in patients; moreover, a prolonged operation time affects organ perfusion. Therefore, longer duration of surgery is associated with unfavorable outcomes.

Echocardiography is an important tool for the diagnosis of ATAAD. There were 188 cases of ATAAD with medium to large amounts of pericardial effusion, and the proportion in the nonsurvivor group was significantly higher than that in the survivor group. Our findings revealed that moderate to severe pericardial effusion was a risk factor for postoperative death in patients with ATAAD. Moderate to severe pericardial effusion is a sign of hemodynamic instability and aortic rupture in ATAAD patients. Santi Trimarchi et al. demonstrated that pericardial tamponade was an independent predictor of postoperative mortality in patients with ATAAD (OR: 2.22)(7). The inner diameter of the ascending aorta of ATAAD patients was obtained by echocardiography and CTA measurements; the width was obviously increased, but the difference between the nonsurvivor group and the survivor group was not statistically significant. A report from the IRAD noted that the vast majority of ATAAD patients had an enhanced ascending aorta diameter, but the mortality in ATAAD patients was independent of the ascending aorta diameter (12). Kim et al. showed that moderate ascending aorta dilatation (inner diameter $<50 \mathrm{~mm}$ ) was moderately correlated with aortic dissection and/or rupture $\left(\mathrm{R}^{2}=0.07\right)(13)$.

Our study revealed that the surgical technique had no significant effect on early postoperative mortality in patients with ATAAD; this finding could be related to selection bias in the surgical method choice. It could also be related to the interaction among various operating parameters. Goda et al. indicated that various surgical methods did not affect the early postoperative mortality rate of ATAAD patients (8). However, Ten et al. found that simultaneous aortic valve replacement or Bentall surgery in ATAAD patients was a 
protective factor against postoperative mortality (11).

Our study demonstrated that the admission blood potassium level in the nonsurvivor group was higher than that in the survivor group. However, multivariable analysis showed that potassium was not a risk factor for postoperative mortality in patients with ATAAD. This finding is inconsistent with the results of Chen et al., who found that the blood potassium level at admission $(3.5-4.5 \mathrm{mmol} / \mathrm{l})$ could be related to the increases in in-hospital mortality and long-term mortality in ATAAD patients (14). Our results might have been due to the small number of cases in the nonsurvivor group.

In this study, the proportion of patients with myocardial ischemia in the nonsurvivor group was greater than that in the survivor group. The proportion of patients with ATAAD combined with myocardial ischemia who underwent CABG was also greater in the nonsurvivor group than in the survivor group. When ATAAD was complicated with myocardial ischemia, chest pain was the main symptom, and coronary heart disease was easily misdiagnosed, resulting in a delayed diagnosis of ATAAD and delayed surgical treatment. When ATAAD affects both the left and right coronary arteries, it can lead to acute and severe myocardial ischemia and a poor prognosis. Santini et al. reported that myocardial ischemia is also a risk factor for in-hospital mortality in patients with ATAAD (15). Therefore, CABG should be performed to improve myocardial blood supply in patients with ATAAD complicated with myocardial ischemia. However, the multifactorial analysis in our study indicated that myocardial ischemia was not a risk factor for postoperative mortality; this result may have been related to the CABG operations in these patients. Myocardial ischemia was improved, and no adverse outcomes occurred during the observation period. Imoto et al. suggested that preoperative coronary stent implantation in ATAAD patients could improve the poor prognosis for such patients (16).

\section{Limitations}

This study was not only a single-center study but also of a retrospective nature with consecutive patients, which may have resulted in patient selection bias; thus, the conclusions need to be further verified by multicenter studies. The number of cases in the nonsurvivor group was small, so the sample size should be further expanded. Generally, it is a weakness that not all important details that might have influenced the patients' outcomes can be collected.

\section{Conclusion}

Lower-extremity ischemia and suprasternal branch involvement have the poorest outcomes. Moderate to severe pericardial effusion may influence outcomes. Shorter duration of surgery is associated with better outcomes.

\section{Funding}

This work was supported by the National Natural Science Foundation of China (Nos. 81401432; 81771851; 81727805).

Conflicts of interest: None declared

\section{References}

1. Mozaffarian D Fau - Benjamin, Emelia J, Benjamin Ej Fau - Go, et al. Heart disease and stroke statistics2016 update: A report from the american heart association. Circulation 2016;133(4):e38-360.

2. Conzelmann LO, Weigang E, Mehlhorn U, et al. Mortality in patients with acute aortic dissection type a: Analysis of pre- and intraoperative risk factors from the german registry for acute aortic dissection type a (geraada). European journal of cardio-thoracic surgery : official journal of the European Association for Cardio-thoracic Surgery 2016;49(2):e44-52.

3. Berretta P, Patel HJ, Gleason TG, et al. Irad experience on surgical type a acute dissection patients: Results and predictors of mortality. Annals of cardiothoracic surgery 2016;5(4):346-351. 
4. Wei J, Chen Z, Zhang H, Sun X, Qian X, Yu C. In-hospital major adverse outcomes of acute type a aortic dissection. European journal of cardio-thoracic surgery : official journal of the European Association for Cardio-thoracic Surgery 2019;55(2):345-350.

5. Conzelmann LO, Hoffmann I, Blettner M, et al. Analysis of risk factors for neurological dysfunction in patients with acute aortic dissection type a: Data from the german registry for acute aortic dissection type a (geraada). European journal of cardio-thoracic surgery : official journal of the European Association for Cardio-thoracic Surgery 2012;42(3):557-565.

6. Furukawa T, Uchida N, Takahashi S, et al. Management of cerebral malperfusion in surgical repair of acute type a aortic dissection. European journal of cardio-thoracic surgery : official journal of the European Association for Cardio-thoracic Surgery 2017;52(2):327-332.

7. Trimarchi S, Nienaber CA, Rampoldi V, et al. Contemporary results of surgery in acute type a aortic dissection: The international registry of acute aortic dissection experience. J Thorac Cardiovasc Surg 2005;129(1):112-122.

8. Goda M, Imoto K, Suzuki S, et al. Risk analysis for hospital mortality in patients with acute type a aortic dissection. The Annals of thoracic surgery 2010;90(4):1246-1250.

9. Uchida K KN, Kasama K, Minami T2 Yasuda S2 Goda M2 Suzuki S, Imoto K, Masuda M. Early reperfusion strategy improves the outcomes of surgery for type a acute aortic dissection with malperfusion. J Thorac Cardiovasc Surg 2018;S0022-5223(18):30355-30356.

10. Preece R, Srivastava V, Akowuah E, Kendall S. Should limb revascularization take priority over dissection repair in type a aortic dissection presenting as isolated acute limb ischaemia. Interactive cardiovascular and thoracic surgery 2017;25(4):643-646.

11. Tan M. Operative risk factors of type a aortic dissection: Analysis of 252 consecutive patients. Cardiovascular Surgery 2003;11(4):277-285.

12. Pape LA, Tsai TT, Isselbacher EM, et al. Aortic diameter $>$ or $=5.5 \mathrm{~cm}$ is not a good predictor of type a aortic dissection: Observations from the international registry of acute aortic dissection (irad). Circulation 2007;116(10):1120-1127.

13. Kim JB, Spotnitz M, Lindsay ME, MacGillivray TE, Isselbacher EM, Sundt TM, 3rd. Risk of aortic dissection in the moderately dilated ascending aorta. J Am Coll Cardiol 2016;68(11):1209-1219.

14. Chen Z, Huang B, Lu H, et al. The effect of admission serum potassium levels on in-hospital and long-term mortality in type a acute aortic dissection. Clinical biochemistry 2017;50(15):843-850.

15. Santini F, Montalbano G, Casali G, et al. Clinical presentation is the main predictor of in-hospital death for patients with acute type a aortic dissection admitted for surgical treatment: A 25 years experience. International journal of cardiology 2007;115(3):305-311.

16. Imoto K UK, Suzuki S, Isoda S, Karube N, Kimura K. Stenting of a left main coronary artery dissection and stent-graft implantation for acute type a aortic dissection. J Endovasc Ther 2005;12(2):258-261.

\section{Hosted file}

Table.pdf available at https://authorea.com/users/413008/articles/521477-clinical-analysisof-risk-factors-for-30-day-mortality-in-type-a-acute-aortic-dissection-a-single-centerstudy-from-china 
Figure 1. ROC curves of varying parameters for 30-day mortality in patients with ATAAD

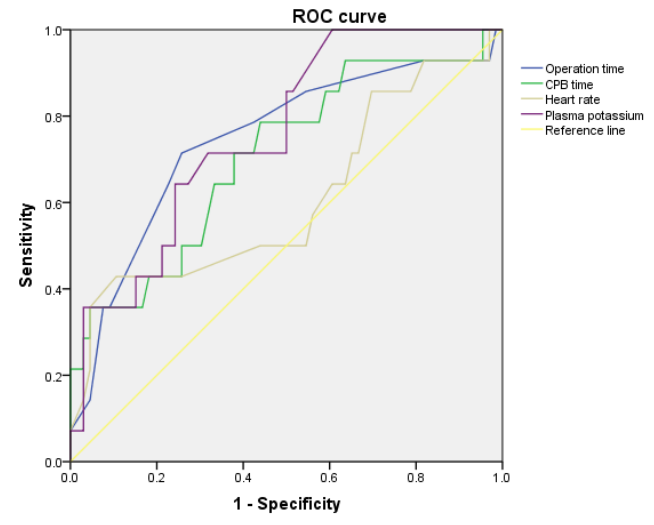

